\title{
Radiation doses, noise properties, and in-plane spatial resolutions for CT coronary angiography using different CT scanners: Phantom study
}

\author{
Kosuke Matsubara ${ }^{1}$, Tadanori Takata ${ }^{2}$, Hiroshige Mori ${ }^{3}$, Masanao Kobayashi ${ }^{4}$, Katsuhiro \\ I chikawa' ${ }^{1}$, Kichiro Koshida ${ }^{1}$
}

1. Department of Quantum Medical Technology, Kanazawa University, Kanazawa, Japan. 2. Department of Radiological Technology, Kanazawa University Hospital, Kanazawa, Japan. 3. Department of Radiology, JCHO Hokkaido Hospital, Toyoake, Japan. 4. Department of Radiology, Fujita Health University Hospital, Toyoake, Japan

Correspondence: Kosuke Matsubara. Address: Department of Quantum Medical Technology, Kanazawa University, Kanazawa, Japan. Email: matsuk@mhs.mp.kanazawa-u.ac.jp

Received: December 17, 2014 Accepted: January 21, $2015 \quad$ Online Published: March 5, 2015

DOI : 10.5430/ijdi.v2n2p1 URL: http://dx.doi.org/10.5430/ijdi.v2n2p1

\section{Abstract}

Objective: We aimed to evaluate radiation doses, noise properties, and in-plane spatial resolutions for CT coronary angiography (CTCA) using different CT scanners and phantoms.

Methods: ECG-gated step-and-shoot (SAS) and low-pitch spiral (LPS) acquisitions were performed using the 64-section single-source CT (SSCT) system. ECG-gated high-pitch spiral (HPS), SAS, and LPS acquisitions were performed using the 128-section dual-source CT (DSCT) system. The absorbed doses for thoracic organs were measured using an anthropomorphic phantom and radiophotoluminescent glass dosimeters. Noise properties and in-plane spatial resolutions were evaluated using a calibration phantom and a cylinder phantom constructed in-house, respectively.

Results: The absorbed doses for thoracic organs differed significantly depending on the acquisition modes and not the scanners. The doses absorbed by the heart were 66.8 and $215.5 \mathrm{mGy}$ with SAS and LPS acquisitions using the 64-section SSCT system, respectively, and 10.0, 96.1, and 195.7 mGy with HPS, SAS, and LPS acquisitions using the 128-section DSCT system, respectively. Noise properties and in-plane spatial resolutions differed significantly depending on the scanners used but not the acquisition modes.

Conclusions: To optimize the patient dose, an appropriate acquisition mode should be chosen according to the scanner type, patient's average heart rate, stability of a patient's heartbeat, and patient's history.

\section{Keywords}

Computed tomography, Coronary angiography, Image quality, Radiation dose

\section{I ntroduction}

The introduction of 64-section multi-detector row CT (MDCT) systems has enabled various types of CT examinations with high spatial and temporal resolutions. CT coronary angiography (CTCA) is a type of CT examination that needs high spatial and temporal resolutions ${ }^{[1]}$. It has evolved as a high accuracy non-invasive tool for assessing coronary artery 
disease. Although some cases of coronary artery stenosis may be misdiagnosed using the 64-section system ${ }^{[2]}$, some reports have demonstrated the effectiveness of this system. Das et al. ${ }^{[3]}$ reported that the 64-section CT system could depict in-stent low-attenuation filling defects, which appear to be a reliable sign of stent restenosis, with a high degree of accuracy. Leschka et al. ${ }^{[4]}$ reported that the depiction rate of myocardial bridging, which is an important anomaly clinically, was greater by 64-section CTCA than by conventional coronary angiography.

Dual-source CT (DSCT), one of the latest improvements in CT technology, can be used for CTCA with twice the temporal resolution while maintaining the spatial resolution of single-source $\mathrm{CT}^{[5]}$. Achenbach et al. ${ }^{[6]}$ reported that heart rate control significantly improved the evaluation capability with single-source CT (SSCT), whereas it did not with DSCT. Second-generation DSCT in which the system is equipped with a 128-section detector has a high temporal resolution of 75 $\mathrm{ms}^{\text {[7] }}$. It can be used for prospective electrocardiogram (ECG)-gated step-and-shoot (SAS) acquisition for patients with high heart rates (> 65 beats per minute; bpm) ${ }^{[8]}$ It can also be used for prospective ECG-gated high-pitch spiral (HPS) acquisition that allows scanning of the heart with a total scan time of approximately $270 \mathrm{~ms}$ with $<1 \mathrm{mSv}{ }^{[9]}$. Neefjes et al. ${ }^{[8]}$ reported that HPS-CTCA should be used for patients with low heart rates $(<55 \mathrm{bpm})$, while SAS-CTCA is preferred for all other cases.

During CTCA, a patient receives a relatively high radiation dose, which has raised concerns regarding radiation exposure $^{[10,11]}$. To perform CTCA with optimal acquisition parameters, it is necessary to evaluate the radiation dose and physical image quality of the scanner used. In this study, we evaluated the radiation doses absorbed by thoracic organs, noise properties, and spatial resolutions for CTCA using different CT scanners and phantoms.

\section{Methods}

\subsection{CT scanner}

For 64-section SSCT and 128-section DSCT, LightSpeed VCT (GE Healthcare, Milwaukee, WI, USA) and SOMATOM Definition Flash (Siemens Healthcare, Erlangen, Germany) were used, respectively.

\subsection{CTCA acquisition modes}

In general, CTCA is performed by applying one of two acquisition modes: retrospective ECG-gated low-pitch spiral (LPS) acquisition or prospective ECG-gated SAS acquisition ${ }^{[12]}$. For LPS acquisition, CT data are acquired throughout the cardiac cycle, and the data required for the reconstruction phase is chosen retrospectively (see Figure 1a). For SAS acquisition, CT data are only acquired over a fraction of the R-R interval (see Figure 1b).

SAS acquisition allows for low radiation exposure during CTCA and provides a high diagnostic accuracy for the assessment of coronary artery disease. Previous studies reported that SAS acquisition effectively reduced radiation doses in CTCA without compromising image quality ${ }^{[13,14]}$. However, it can only be used for in patients with stable sinus rhythms and low heart rates ${ }^{[15]}$. In contrast, the LPS mode can be used for patients with high heart rates; however, the radiation doses received by patients are considerably high because a low spiral pitch is chosen ${ }^{[15]}$.

Because of its high temporal resolution, 128-slice DSCT can be used for prospective ECG-gated HPS acquisition that allows scanning of the heart during a single heartbeat (see Figure 1c). With SSCT, sampling gaps, which are the causes of wide slice sensitivity profiles and image artifacts, occur when the spiral pitch is $>1.5$. With 128-section DSCT, however, data acquired using the second x-ray tube and detector can be used to fill these gaps, and the spiral pitch can be increased up to 3.4. By using a spiral pitch of 3.4, it can also scan the heart in a total time of approximately $270 \mathrm{~ms}{ }^{[9]}$. 
(a)

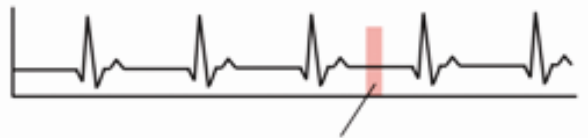

High-pitch spiral acquisition

(b)

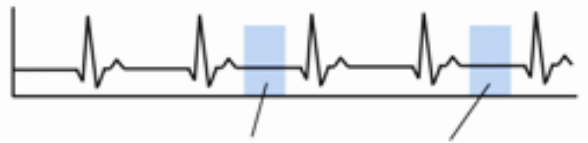

Step-and-shoot acquisition

(c)

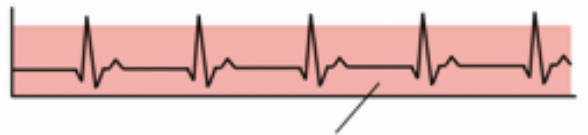

Low-pitch spiral acquisition

Figure 1. Illustration of the concept of three acquisition modes in CTCA: (a) prospective ECG-gated HPS acquisition; (b) prospective ECG-gated SAS acquisition, and (c) retrospective ECG-gated LPS acquisition

\subsection{Comparison of absorbed doses for thoracic organs}

An anthropomorphic RANDO phantom (RAN-110; Phantom Laboratory, Salem, NY, USA; see Figure 2) and radiophotoluminescent glass dosimeters (RPLDs) (GD-302M; Chiyoda Technol, Tokyo, Japan) were used to measure radiation doses absorbed by thoracic organs. Dose calibration of RPLDs was performed against an ionizing dosimeter (Ramtec 1500B; Toyo Medic, Tokyo, Japan) using a $3-\mathrm{cm}^{3}$ ion chamber attached to a $120-\mathrm{kVp}$ diagnostic $\mathrm{x}$-ray beam (effective energy $=50 \mathrm{keV}$ ). The ionizing dosimeter was calibrated prior to use.

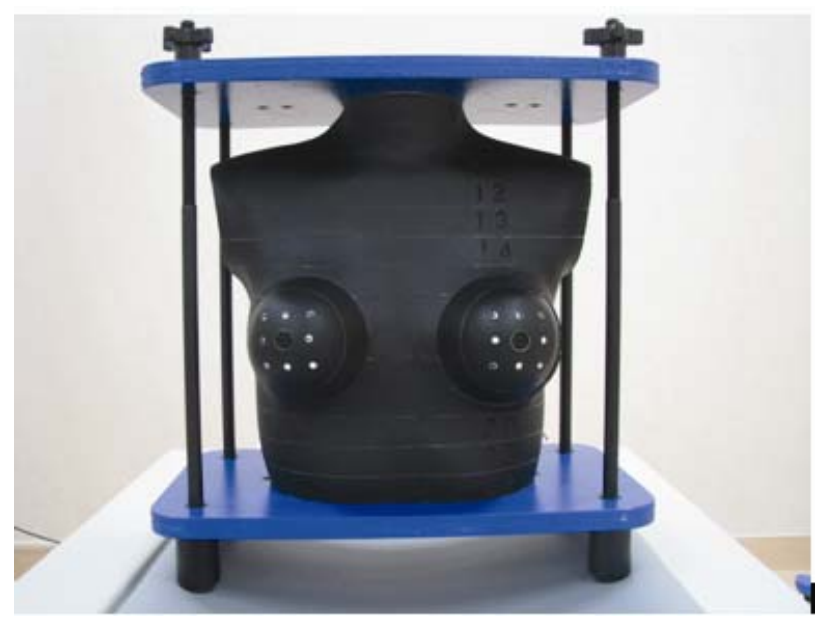

Figure 2. An anthropomorphic female thoracic RANDO phantom. The phantom has multiple transverse sections, and radiophotoluminescent glass dosimeters can be inserted into the sections

RPLDs were annealed at $400^{\circ} \mathrm{C}$ for 30 min before use. Locations and numbers of RPLDs are shown in Table 1 . The RPLDs were evenly distributed at locations that corresponded to those of each organ. Subsequently, SAS (SnapShot Pulse) and LPS acquisitions at a heart rate of $60 \mathrm{bpm}$ were performed using the 64-section SSCT system. ECG-gated HPS (Flash Cardio Spiral), SAS (Flash Cardio Sequence), and LPS acquisitions at a heart rate of $60 \mathrm{bpm}$ were performed using the 128-section DSCT system. Exposure parameters for radiation dose measurements are shown in Table 2. To achieve accurate RPLD readings, the measurement was repeated twice using separate sets of RPLDs. 
After each exposure, RPLDs were further heated to $70^{\circ} \mathrm{C}$ for $30 \mathrm{~min}$ and were read using a FGD-1000 reader (Chiyoda Technol) in accordance with the manufacturer's recommended protocol. The absorbed organ doses were obtained by multiplying the averaged dose value of the organ obtained from the reader, calibration factor, and the ratio of mass energy-absorption coefficients for each organ to air ${ }^{[16]}$.

Table 1. Locations and numbers of radiophotoluminescent glass dosimeters

\begin{tabular}{ll}
\hline Location & Number of dosimeters \\
\hline Breast & 6 \\
Heart & 8 \\
Lung & 8 \\
Red bone marrow (ribs) & 6 \\
Red bone marrow (sternum) & 2 \\
Red bone marrow (thoracic vertebrae) & 4 \\
Thymus & 2 \\
Skin & 12 \\
Background & 2 \\
Total & 50 \\
\hline
\end{tabular}

Table 2. Exposure and reconstruction parameters for measuring absorbed doses for thoracic organs and for evaluating physical image quality

\begin{tabular}{|c|c|c|c|c|c|}
\hline \multirow{2}{*}{ Parameters } & \multicolumn{5}{|c|}{ Acquisition mode } \\
\hline & 64-SSCT SAS & 64-SSCT LPS & 128-DSCT HPS & 128-DSCT SAS & 128-DSCT LPS \\
\hline Collimation (mm) & $64 \times 0.625$ & $64 \times 0.625$ & $128 \times 0.6$ & $128 \times 0.6$ & $128 \times 0.6$ \\
\hline $\mathrm{kV}$ & 120 & 120 & 120 & 120 & 120 \\
\hline Spiral pitch & N/A & $0.16: 1$ & $3.4: 1$ & N/A & $0.17: 1$ \\
\hline $\mathrm{mAs}$ & 245 & 245 & 340 & 340 & 340 \\
\hline Rotation time (s) & 0.35 & 0.35 & 0.28 & 0.28 & 0.28 \\
\hline Scan range (mm) & 175 & 150 & 153 & 172 & 153 \\
\hline Padding window* & $200 \mathrm{~ms}$ & N/A & N/A & $35 \%-85 \%$ & N/A \\
\hline Slice thickness (mm) & 0.625 & 0.625 & 0.75 & 0.75 & 0.75 \\
\hline Kernel & Standard & Standard & B35f & B35f & B35f \\
\hline CTDIvol (mGy) & 37.45 & 114.22 & 5.49 & 51.32 & 110.21 \\
\hline
\end{tabular}

Note. *Prolonging window in data acquisition to adapt heart rate variation.

64-SSCT = 64-section single-source computed tomography; 128-DSCT = 128-section dual-source computed tomography; SAS = step-and-shoot; LPS = low-pitch spiral; HPS = high-pitch spiral; N/A = not applicable.

\subsection{Comparison of noise properties}

A calibration phantom (Catphan 600; Phantom Laboratory), which was attached a part \#579, was used to evaluate noise properties (see Figure 3). The Catphan 600 calibration phantom consisted of five modules: CTP 404, 486, 515, 528, and 591. The reconstructed images for CTP 486 (image uniformity module), which were scanned at the isocenter, were used $^{[17]}$. All images were reconstructed with a displayed field of view (DFOV) of $200 \mathrm{~mm}$. Exposure and reconstruction parameters were the same as those shown in Table 2.

To calculate a noise power spectrum (NPS), the central $256 \times 150$ pixels in the reconstructed images were used. One-dimensional NPS was calculated using a Fourier transform of one-dimensional noise profiles obtained using the numerical slit scanning technique ${ }^{[17]}$. In this technique, five slits each with a height of 30 pixels $(1 \times 30$ pixels $)$ were used. To improve the accuracy of NPS data, an average NPS was calculated using five different CT images. 


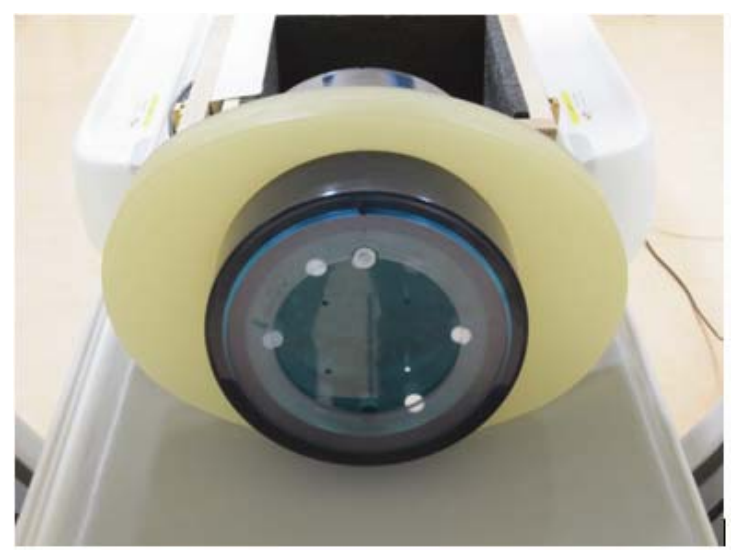

Figure 3. A calibration phantom with an attached elliptical-shaped part. Using an elliptical-shaped phantom is a better choice for evaluating noise properties because the images obtained with a circular-shaped phantom may be biased

\subsection{Comparison of in-plane spatial resolutions}

A cylinder phantom constructed in-house was used to evaluate in-plane spatial resolutions (see Figure 4) ${ }^{[18]}$. The phantom was positioned $20 \mathrm{~mm}$ above the isocenter of the gantry along the y-axis. To avoid aliasing errors, images were reconstructed so that the wire was located at the center of the images with a DFOV of $100 \mathrm{~mm}$ (sampling pitch was $0.196 \mathrm{~mm}$ ). Exposure and reconstruction parameters were the same as those shown in Table 2.

To calculate an in-plane modulation transfer function (MTF), a slit with a height of 40 pixels $(1 \times 40$ pixels) was used to obtain one-dimensional profiles that were equivalent to a line spread function (LSF). Subsequently, the in-plane MTF was calculated using one-dimensional Fourier transformations of LSF. To improve the accuracy of MTF data, the average MTF was calculated using three different CT images.

(a)

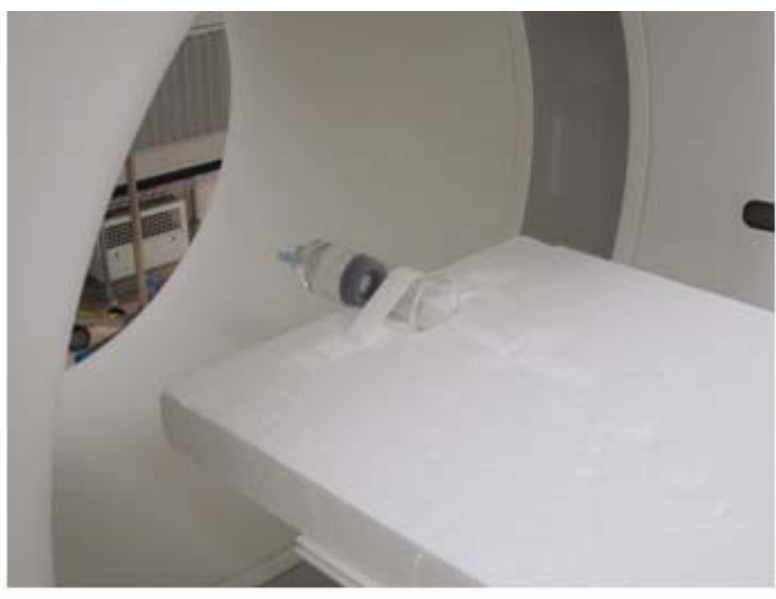

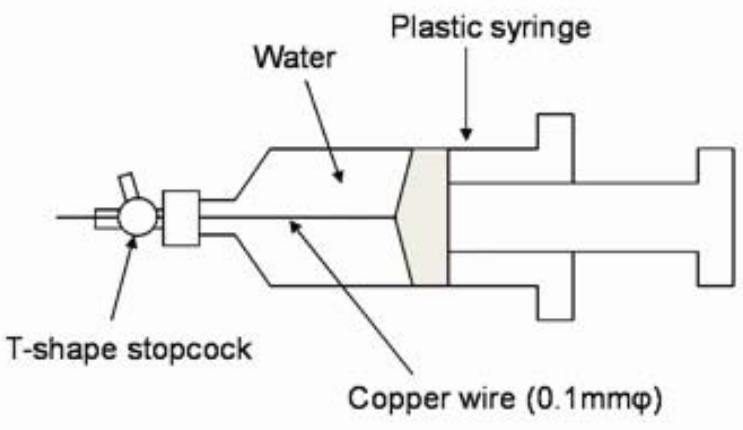

(b)

Figure 4. A cylinder phantom constructed in-house for evaluation of in-plane spatial resolutions. (a) the arrangement of the phantom and (b) the construction of the phantom. The cylinder phantom was filled with water and had a diameter of $50 \mathrm{~mm}$. A copper wire with a diameter of $0.1 \mathrm{~mm}$ was stretched in the center of the phantom

\subsection{Software}

ImageJ (National Institutes of Health, Bethesda, MD, USA) and Excel 2010 (Microsoft, Redmond, WA, USA) were used to evaluate the physical image quality. 


\section{Results}

\subsection{Comparison of absorbed doses for thoracic organs}

Figure 5 shows the results of the doses absorbed by each organ according to the scanners used and the acquisition modes at a fixed heart rate of $60 \mathrm{bpm}$. These results showed that the absorbed doses for thoracic organs differed significantly depending on the acquisition modes but not the scanners. However, they differed even if the same acquisition mode was selected with different scanners because the spiral pitch or padding window differed between the scanners used.

In the results shown in Figure 5, the doses absorbed by the heart were the highest; 66.8 and 215.5 mGy with SAS and LPS acquisitions using the 64-section SSCT system, respectively, and 10.0, 96.1, and 195.7 mGy with HPS, SAS, and LPS acquisitions using the 128-section DSCT system, respectively. The doses absorbed by the breast were approximately half those absorbed by the heart.

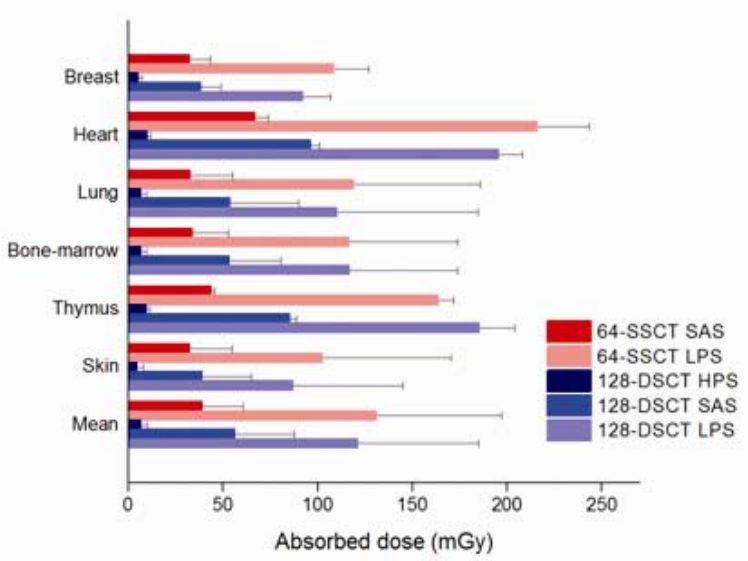

Figure 5. Differences in absorbed doses for each organ according to the scanners used and the acquisition modes at a fixed heart rate of $60 \mathrm{bpm}$

\subsection{Comparison of noise properties}

Figure 6 shows the results of NPS values according to the scanners used and the acquisition modes. NPS values of $<0.7$ cycles/mm with LPS acquisition were superior to those with other acquisitions, and NPS values of $>0.7$ cycles/mm with LPS acquisition were inferior to those with other acquisitions using the 128-section DSCT system. However, noise properties differed significantly depending on the scanners used.

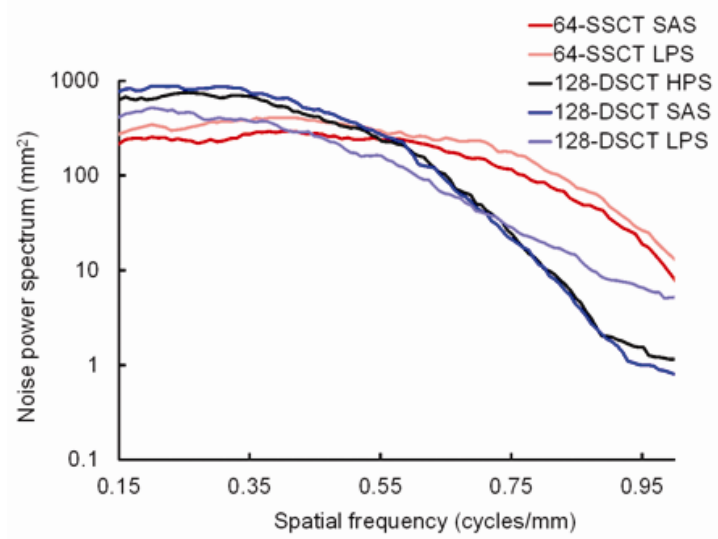

Figure 6. Differences in NPS values according to the scanners used and the acquisition modes 


\subsection{Comparison of in-plane spatial resolutions}

Figure 7 shows the results of in-plane MTF according to the scanners used and the acquisition modes. The values for $50 \%$ MTF were 0.44 and 0.42 with SAS and LPS acquisitions using the 64-section SSCT system, respectively, and the values were $0.31,0.34$, and 0.34 with HPS, SAS, and LPS acquisitions using the 128-section DSCT system, respectively. The values for $10 \%$ MTF were 0.69 and 0.68 with SAS and LPS acquisitions using the 64-section SSCT system, respectively, and the values were 0.59, 0.59, and 0.59 with HPS, SAS and LPS acquisitions using the 128-section DSCT system, respectively. In-plane spatial resolutions also differed significantly depending on the scanners used.

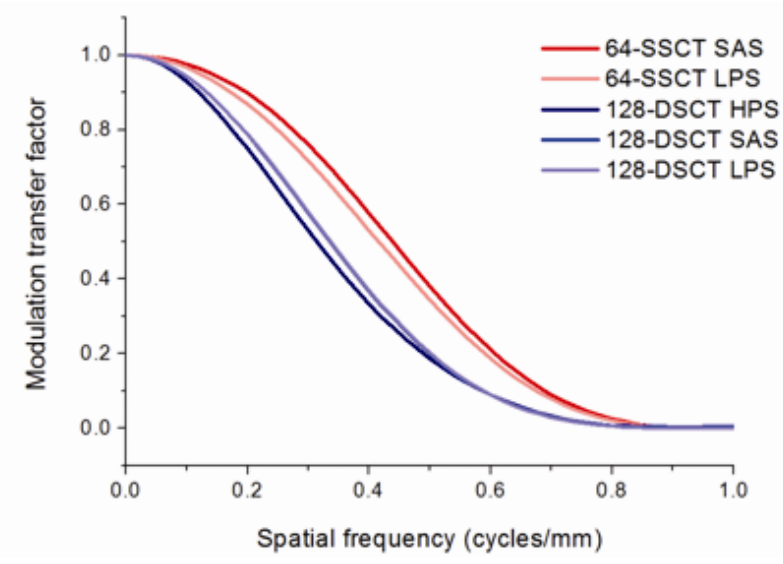

Figure 7. Differences in in-plane MTF according to the scanners used and the acquisition modes

\section{Discussion}

In this study, we evaluated absorbed doses for thoracic organs, noise properties, and in-plane spatial resolutions for CTCA using two different CT scanners. The absorbed doses for thoracic organs differed significantly depending on the acquisition modes but not the scanners. In contrast, although physical image quality, such as noise properties and in-plane spatial resolutions, differed slightly depending on the acquisition modes, it differed significantly depending on the scanners used, especially the reconstruction kernel that was used.

The 128-section DSCT system had an advantage in that we could apply a dose-saving acquisition mode, such as HPS acquisition, for patients with relatively high heart rates. Srichai et al. ${ }^{[19]}$ reported that HPS acquisition had image quality no worse than that of LPS acquisition, and there was no significant difference in the inter-reader variability in diagnosis between the HPS and LPS acquisitions. However, they excluded patients who had a history of coronary artery bypass surgery or metallic prosthetic valve replacement surgery from the study population. In our previous study, the number of artifacts in the reconstructed image for the HPS acquisition was larger than those for the SAS and LPS acquisitions ${ }^{[20]}$. It is important that we should choose an appropriate acquisition mode according to the scanner type, patient's average heart rate, stability of a patient's heartbeat, and patient's history to optimize the patient dose.

The radiation doses absorbed by thoracic organs are somewhat different depending on the scanner, even if the same acquisition mode is chosen because the spiral pitch or padding window is different between scanners. The radiation dose can be reduced when the spiral pitch is increased or the padding window is narrowed. Therefore, this is effective for dose saving that can be adjusted in addition to selecting an appropriate acquisition mode.

Of course, adjusting the tube current is also effective for dose saving. Recently, some scanners have been used for CTCA by using the tube current modulation (TCM) technique. May et al. ${ }^{[21]}$ reported that radiation dose savings up to $52 \%$ were 
achievable by TCM at low and regular HR. Scanners for which a TCM technique cannot be used for CTCA, we should adjust the tube current manually based on patient size.

If images having good noise properties are needed, choosing a smooth reconstruction kernel is the most effective rather than increasing the tube current or selecting other acquisition modes. It is also effective to apply an iterative reconstruction algorithm. Some previous study reported that the iterative reconstruction algorithm significantly reduced image noise and yielded a higher contrast-to-noise ratio of coronary artery ${ }^{[22,23]}$. Takata et al. ${ }^{[24]}$ also demonstrated that the iterative reconstruction algorithm had the noise reduction performance with exact preservation for high contrast resolution and slight degradation of middle contrast resolution. However, generally noise properties and in-plane spatial resolutions have a trade-off relationship. Our results showed that images obtained using the 64-section SSCT system had better in-plane spatial resolutions than those obtained using the 128-section DSCT system, while those obtained using the 128-section DSCT system had better noise properties than those obtained using the 64-section SSCT system. It is almost impossible to identify one type of physical image quality with another, although we need to choose an appropriate reconstruction kernel to improve diagnostic capability through evaluation of physical image quality.

Due to the experimental design of the study, our results cannot be directly transferred into the clinical situation. For example, it is well known that cardiac motion artifacts can degrade the diagnostic accuracy and interpretability of coronary artery disease in CTCA ${ }^{[25,26]}$. In this study, however, physical image quality of only motion artifact-free images was evaluated. Penzkofer et al. ${ }^{[27]}$ used a cardiac motion phantom and showed that motion artifacts were present in the $90 \mathrm{bpm}$ HPS acquisition, which were not present in the LPS acquisition or the 50 or $70 \mathrm{bpm}$ HPS acquisition. Therefore, we believe selecting appropriate acquisition mode and reconstruction kernel are both important for improving diagnostic capability.

There were some limitations of our study. First, we used one specific female anthropomorphic phantom. The absorbed dose for each organ differed according to the size and shape of each phantom. Second, although we evenly distributed RPLDs at locations that corresponded to those of each organ, a limited number of RPLDs caused the uncertainty of obtained organ doses. Third, we only simulated a heart rate of $60 \mathrm{bpm}$, and hence, no data was available for other heart rates. Finally, we only evaluated radiation dose and physical image quality (NPS and in-plane MTF) of the scanners used. We think observer-performance studies regarding phantom and clinical images will also be needed to evaluate comprehensive image quality.

\section{Conclusion}

The absorbed doses for thoracic organs differ significantly depending on the acquisition modes but not the scanners used. In contrast, noise properties and in-plane spatial resolutions differ significantly depending on the scanners used but not the acquisition modes. One should choose an appropriate acquisition mode according to the scanner type, patient's average heart rate, stability of a patient's heartbeat, and patient's history to optimize patient dose, and choose appropriate acquisition mode and reconstruction kernel to improve diagnostic capability.

\section{Acknowledgement}

This work was supported by MEXT KAKENHI Grant Number 21791175.

\section{References}

[1] Donnelly PM, Higginson JD, Hanley PD. Multidetector CT coronary angiography: have we found the holy grail of non-invasive coronary imaging? Heart. 2005; 91: 1385-1388. PMid:16230434 http://dx.doi.org/10.1136/hrt.2004.058164 
[2] Gouya H, Varenne O, Trinquart L, et al. Coronary artery stenosis in high-risk patients: 64-section CT and coronary angiography prospective study and analysis of discordance. Radiology. 2009; 252: 377-385. PMid:19546426 http://dx.doi.org/10.1148/radiol.2522081271

[3] Das KM, El-Menyar AA, Salam AM, et al. Contrast-enhanced 64-section coronary multidetector CT angiography versus conventional coronary angiography for stent assessment. Radiology. 2007; 245: 424-432. PMid:17890354 http://dx.doi.org/10.1148/radiol.2452061389

[4] Leschka S, Koepfli P, Husmann L, et al. Myocardial bridging: depiction rate and morphology at CT coronary angiographycomparison with conventional coronary angiography. Radiology. 2008; 246: 754-762. PMid:18223120 http://dx.doi.org/10.1148/radiol.2463062071

[5] Flohr TG, McCollough CH, Bruder H, et al. First performance evaluation of a dual-source CT (DSCT) system. Eur Radiol. 2006; 16: 256-268. http://dx.doi.org/10.1007/s00330-005-2919-2

[6] Achenbach S, Ropers U, Kuettner A, et al. Randomized comparison of 64-slice single and dual-source computed tomography coronary angiography for the detection of coronary artery disease. JACC Cardiovasc Imaging. 2008; 1: 177-186. PMid:19356426 http://dx.doi.org/10.1016/j.jcmg.2007.11.006

[7] Sommer WH, Albrecht E, Bamberg F, et al. Feasibility and radiation dose of high-pitch acquisition protocols in patients undergoing dual-source cardiac CT. AJR Am J Roentgenol. 2010; 195: 1306-1312. PMid:21098188 http://dx.doi.org/10.2214/AJR.10.4416

[8] Neefjes LA, Dharampal AS, Rossi A, et al. Image quality and radiation exposure using different low-dose scan protocols in dual-source CT coronary angiography: randomized study. Radiology. 2011; 261: 779-786. PMid:21969666 http://dx.doi.org/10.1148/radiol.11110606

[9] Achenbach S, Marwan M, Ropers D, et al. Coronary computed tomography angiography with a consistent dose below $1 \mathrm{mSv}$ using prospectively electrocardiogram-triggered high-pitch spiral acquisition. Eur Heart J. 2010; 31: 340-346. PMid:19897497 http://dx.doi.org/10.1093/eurheartj/ehp470

[10] Einstein AJ, Henzlova MJ, Rajagopalan S. Estimating risk of cancer associated with radiation exposure from 64-slice computed tomography coronary angiography. JAMA. 2007; 298: 317-323. PMid:17635892 http://dx.doi.org/10.1001/jama.298.3.317

[11] Gerber TC, Carr JJ, Arai AE, et al. Ionizing radiation in cardiac imaging: a science advisory from the American Heart Association Committee on Cardiac Imaging of the Council on Clinical Cardiology and Committee on Cardiovascular Imaging and Intervention of the Council on Cardiovascular Radiology and Intervention. Circulation. 2009; 119: 1056-1065. PMid:19188512 http://dx.doi.org/10.1161/CIRCULATIONAHA.108.191650

[12] Steigner ML, Otero HJ, Cai T, et al. Narrowing the phase window width in prospectively ECG-gated single heart beat 320-detector row coronary CT angiography. Int J Cardiovasc Imaging. 2009; 25: 85-90. PMid:18663599 http://dx.doi.org/10.1007/s10554-008-9347-8

[13] Ko SM, Kim NR, Kim DH, et al. Assessment of image quality and radiation dose in prospective ECG-triggered coronary CT angiography compared with retrospective ECG-gated coronary CT angiography. Int J Cardiovasc Imaging. 2010; 26: 93-101. PMid:20033490 http://dx.doi.org/10.1007/s10554-009-9554-y

[14] Efstathopoulos EP, Kelekis NL, Pantos I, et al. Reduction of the estimated radiation dose and associated patient risk with prospective ECG-gated 256-slice CT coronary angiography. Phys Med Biol. 2009; 54: 5209-5222. PMid:19671974 http://dx.doi.org/10.1088/0031-9155/54/17/009

[15] Herzog BA, Husmann L, Burkhard N, et al. Accuracy of low-dose computed tomography coronary angiography using prospective electrocardiogram-triggering: first clinical experience. Eur Heart J. 2008; 29: 3037-3042. PMid:18996954 http://dx.doi.org/10.1093/eurheartj/ehn485

[16] International Commission on Radiation Units and Measurements (ICRU). Tissue substitutes in radiation dosimetry and measurement. ICRU Report 44. Bethesda: ICRU; 1989:1-189.

[17] Giger ML, Doi K, Metz CE. Investigation of basic imaging properties in digital radiography. 2. noise wiener spectrum. Med Phys. 1984; 11: 797-805. PMid:6513887 http://dx.doi.org/10.1118/1.595583

[18] Ichikawa K, Hara T, Niwa S, et al. Method of measuring modulation transfer function using metal wire in computed tomography. Nihon Hoshasen Gijutsu Gakkai Zasshi. 2008; 64: 672-680.

[19] Srichai MB, Lim RP, Donnino R, et al. Low-dose, prospective triggered high-pitch spiral coronary computed tomography angiography: comparison with retrospective spiral technique. Acad Radiol. 2012; 19: 554-561. PMid:22366557 http://dx.doi.org/10.1016/j.acra.2012.01.009

[20] Matsubara K, Koshida H, Sakuta K, et al. Radiation dose and physical image quality in 128-section dual-source computed tomographic coronary angiography: a phantom study. J Appl Clin Med Phys. 2012; 13: 252-261. 
[21] May MS, Deak P, Kuettner A, et al. Radiation dose considerations by intra-individual Monte Carlo simulations in dual source spiral coronary computed tomography angiography with electrocardiogram-triggered tube current modulation and adaptive pitch. Eur Radiol. 2012; 22: 569-578. PMid:21984448 http://dx.doi.org/10.1007/s00330-011-2300-6

[22] Scheffel H, Stolzmann P, Schlett CL, et al. Coronary artery plaques: cardiac CT with model-based and adaptive-statistical iterative reconstruction technique. Eur J Radiol. 2012; 81: e363-369. PMid:22197733 http://dx.doi.org/10.1016/j.ejrad.2011.11.051

[23] Utsunomiya D, Weigold WG, Weissman G, et al. Effect of hybrid iterative reconstruction technique on quantitative and qualitative image analysis at 256-slice prospective gating cardiac CT. Eur Radiol. 2012; 22: 1287-1294. PMid:22200900 http://dx.doi.org/10.1007/s00330-011-2361-6

[24] Takata T, Ichikawa K, Hayashi H, et al. Image quality evaluation of new image reconstruction methods applying the iterative reconstruction. Nihon Hoshasen Gijutsu Gakkai Zasshi. 2012; 68: 404-412.

[25] Kroft LJ, de Roos A, Geleijns J. Artifacts in ECG-synchronized MDCT coronary angiography. AJR Am J Roentgenol. 2007; 189: 581-591. PMid:17715104 http://dx.doi.org/10.2214/AJR.07.2138

[26] Leipsic J, Labounty TM, Hague CJ, et al. Effect of a novel vendor-specific motion-correction algorithm on image quality and diagnostic accuracy in persons undergoing coronary CT angiography without rate-control medications. J Cardiovasc Comput Tomogr. 2012; 6: 164-71. PMid:22551593 http://dx.doi.org/10.1016/j.jcct.2012.04.004

[27] Penzkofer T, Donandt E, Isfort P, et al. Influence of trigger type, tube voltage and heart rate on calcified plaque imaging in dual source cardiac computed tomography: phantom study. BMC Med Imaging. 2014; 14: 30. PMid:25178653

http://dx.doi.org/10.1186/1471-2342-14-30 